\title{
Study of membrane defects induced by antimicrobial and hemolytic peptide Ltc1 in erythrocyte membrane
}

\author{
Nikita Orlov ${ }^{1}$, Olga Geraskina ${ }^{2}$ and Alexey Feofanov ${ }^{3}$ \\ ${ }^{1}$ Lomonosov Moscow State University, Moscow, Moskva, Russia, ${ }^{2}$ Lomonosov Moscow State University, \\ Russia, ${ }^{3}$ Biological Faculty, Lomonosov Moscow State University, Moscow, Russia, Moscow, Russia \\ *corresponding author: n.orlov858@yandex.ru
}

The antimicrobial resistance is a global challenge driving a search for alternatives to classical antibiotics. Naturally occurring antimicrobial peptides are considered as promising candidates for fighting multi-resistant bacteria, but their therapeutic potential is strictly dependent on their safety to host cells. Latarcin Ltc1 is a spider venom antimicrobial peptide, which is linear, short (25 a.a.), cationic (net charge +9 at physiological $\mathrm{pH}$ ) and undergoes coil-to- $\alpha$-helix transition in membrane-mimicking environment [1]. In addition to high antimicrobial activity Ltc1 possesses cytotoxic and hemolytic action, which requires detailed investigation. Earlier it was shown that hemolytic activity of non-processed form of Ltc1 (i.e. Ltc1-K) is regulated by its Nterminal moiety [2]. Here we report on the features of interactions of mature Ltc1 with human red blood cells revealed with a confocal laser scanning microscopy (CLSM).

For CLSM studies, synthetic Ltc1 tagged with sulforhodamine B (Rh-Ltc1) was used. Comparative analysis of Ltc1 and Rh-Ltc1 activities in the RPMI-1640 culture medium revealed that their effective hemolytic concentrations $\left(\mathrm{EC}_{50}\right)$ were $1.0 \pm 0.1$ and $0.4 \pm 0.1 \mu \mathrm{M}$, respectively. Therefore, hemolytic activity of Ltc1 was not impaired by the fluorescent label. Real time study of human erythrocyte hemolysis revealed a long phase of Rh-Ltc1 accumulation in erythrocyte membrane accompanied by erythrocyte crenation according to the pathway of discocyte - echinocyte - stomatocytes - spherocyte (Fig. 1, A, B). This phase was followed by a fast hemoglobin $(\mathrm{Hb})$ release through the formed membrane openings and, finally, by a ghost formation (Fig. 1, A, B). At $3 \mu \mathrm{M}$ Rh-Ltc1, the first phase lasts for tens of minutes, while Hb release lasts for tens of seconds. Applying the calibration-based qualitative analysis of CLSM images, membrane density of Rh-Ltc1 was estimated to be $1200 \pm 200$ molecules $/ \mu \mathrm{m}^{2}$ at the beginning of the first phase and, it raised to $11000 \pm 900$ molecules $/ \mu \mathrm{m}^{2}$ in ghosts. The spherocyte to ghost transformation rate allows one to suppose that Hb leakage occurs through a few membrane openings. Evidently, a diameter of membrane pores during hemolysis is higher than hydrodynamic radius $(\mathrm{HR})$ of $\mathrm{Hb}(\mathrm{ca} .3 .1 \mathrm{~nm})$. But it is not clear, if these pores are transient or stable, and if they are preserved in ghosts.

To evaluate a size of pores, we used the size-marker influx assay described previously [2, 3]. Water-soluble 5carboxyfluorescein (CF) or FITC-labeled dextrans of 10, 70, 250 or 500 kDa (FD10, FD70, FD250, FD500) were added to Rh-Ltc1-produced ghosts, and the degree of their influx was analyzed with CLSM. Size-markers enter into the ghosts indicating preservation of Rh-Ltc1-induced membrane pores. A size of these pores depends on the Rh-Ltc1 concentration added to erythrocytes. At high Rh-Ltc1 concentration (10 $\mu \mathrm{M})$ FD500 (HR 16 nm) fills in 98.5\% ghosts (Fig.2, A) revealing existence of large pores ( $>32 \mathrm{~nm}$ in diameter). At low Rh-Ltc1 concentration $(3 \mu \mathrm{M})$, FD250 (HR 11.5 nm) influx is hampered, CF (HR 1 nm) fills into all ghosts, whereas FD10 (HR 2.3 nm) and FD70 (HR 6 nm) have restricted penetration into ghosts (Fig. 1, C; Fig. 2, B). Accordingly, two populations of ghosts were revealed having diameter of stable pores either 12-23 nm or less than $5 \mathrm{~nm}$. A fraction of ghosts with pores, which are smaller than the $\mathrm{Hb}$ size, indicates that a pore size changes in the course of hemolysis because of alterations in the membrane tension and relaxes to the steady state after $\mathrm{Hb}$ leakage.

Real time imaging of erythrocytes pre-loaded with BCECF-AM and treated with $1 \mu \mathrm{M}$ Rh-Ltc1 didn't reveal any BCECF efflux before $\mathrm{Hb}$ leakage (data not shown), thus rejecting the assumption that $\mathrm{Hb}$ release is preceded by small organic molecules leakage. 
Ltc1 is concluded to induce hemolysis through the formation of stable lipid-peptide pores in the erythrocyte membrane. On the basis of the experimental results and the theory of transient and stable pores in lipid membranes, a model of Ltc1-induced hemolysis is proposed [4].

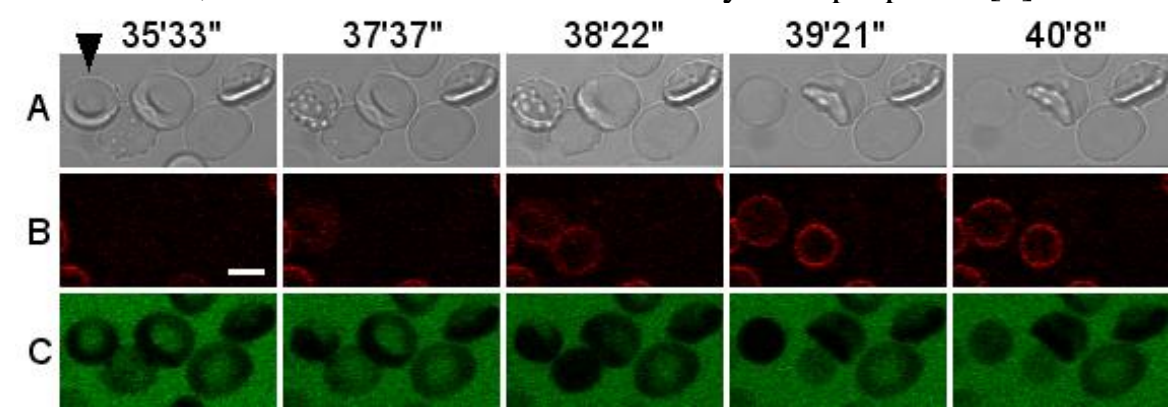

Figure 1. Time-resolved CLSM images of human erythrocytes treated with $3 \mu \mathrm{M}$ Rh-Ltc1 and $10 \mu \mathrm{M}$ FD70 (selected time points are top labeled). Row A - transmitted light images, rows B and C correspond to fluorescence of Rh-Ltc1 and FD70, respectively. Arrow indicates the representative erythrocyte that transforms from a discocyte to a ghost during the time of experiment. Bar - $5 \square \mathrm{m}$.

A

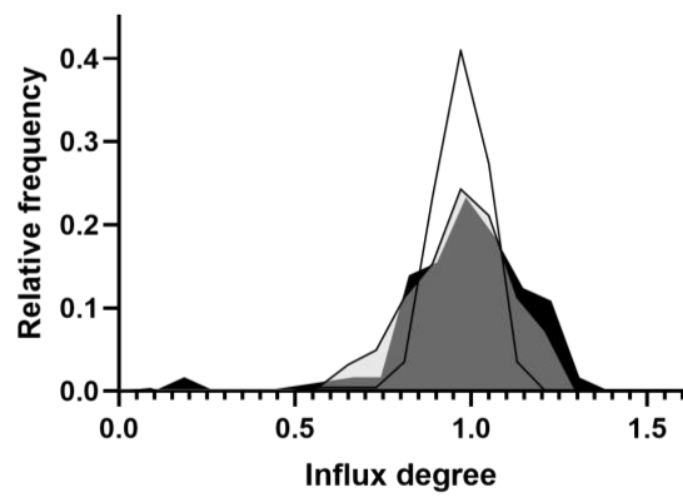

B

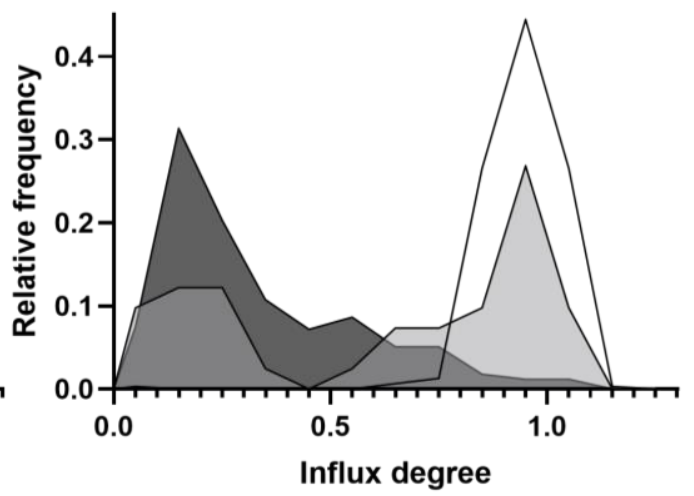

Figure 2. Frequency distributions of Rh-Ltc1-produced ghosts by the size-marker influx degree. Influx degree is the ratio of intracellular to extracellular fluorescence intensity of the size-marker. Concentration of Rh-Ltc1 was $10 \square \mathrm{M}$ (A) or $3 \square \mathrm{M}$ (B). The color scheme used: CF - transparent, FD70 - light gray, FD250 - dark gray, FD500 - black.

\section{References}

[1] Kozlov SA et al. J. Biol. Chem. 281 (2006), p.20983-20992.

[2] Samsonova OV, Kudryashova KS, Feofanov AV. Acta Naturae. 3(2) (2011), p.68-78.

[3] Vorontsova OV et al. Biochimie. 93(2) (2011), p.227-241.

[4] The research was partially performed using facilities of the Interdisciplinary Scientific and Educational School of Moscow State University «Molecular Technologies of the Living Systems and Synthetic Biology». 